\title{
TRIZ Theory and the Method of Cancer Document Selection for Chemical Complexes and Innovation Schemes of Meta-Analysis with Lymphomas as an Example
}

\author{
Yan Huiquan, ${ }^{1,2}$ Lyu Penghui $\mathbb{D}^{2}{ }^{2}$ Wang Ling, ${ }^{3}$ and Yu Zhiming ${ }^{4}$ \\ ${ }^{1}$ Novelty Retrieving Center of National Education Department, Hefei University of Technology, Hefei, Anhui, China \\ ${ }^{2}$ School of Management, Hefei University of Technology, Hefei, Anhui, China \\ ${ }^{3}$ No. 24 Patient Ward, The First Affiliated Hospital of Anhui University of Chinese Medicine, Hefei, China \\ ${ }^{4}$ Department of Biological and Environmental Sciences, Hefei University, Hefei, China
}

Correspondence should be addressed to Lyu Penghui; lyu@hfut.edu.cn

Received 12 October 2019; Accepted 28 February 2020; Published 22 May 2020

Academic Editor: Jia-Bao Liu

Copyright (C) 2020 Yan Huiquan et al. This is an open access article distributed under the Creative Commons Attribution License, which permits unrestricted use, distribution, and reproduction in any medium, provided the original work is properly cited.

In the face of the growing incidence of malignant tumors (about 3.929 million, data issued in January 2019) and the death rate (about 2.338 million, data issued in January 2019) and the limitation of the application of informatics in cancer treatment, this paper tried to use TRIZ theory to deduce new ideas about cancer treatments, perform literature analysis on schemes, and make retrieval strategy for meta-analyses on cancer therapy. By using TRIZ theory and information to analyze the fields of cancers, the research schemes for selecting documents on cancer therapy were presented. After retrieving the documents, we exported all those articles in text format. We further analyzed the research status with the software CiteSpace and Bibliographic Information Mining System (BICOMS) by using different keywords, regions, countries, schools, authors, geography, institutes, etc. We also performed the cluster analysis by using Statistical Package for the Social Sciences (SPSS) software and performed two-way cluster analysis by using Gluto software. The hot areas of research and their tendency or distribution were analyzed. The search strategy was set and the retrieving results were tried.

\section{Introduction to TRIZ}

1.1. The Origin of TRIZ and Its Definition. TRIZ theory provided systematic theoretical and methodological tools for finding and solving problems creatively. TRIZ theory was the theory of inventive problem solving and was developed by the Soviet engineer Genrich Altshuller and his colleagues in 1946. Altshuller and his colleagues studied more than 300,000 patents and developed inventive principles which had been often presented in the most successful cases. According to TRIZ, universal principles of creativity form the basis of innovation. TRIZ identified and codified these principles and used them to make the creative process more predictable. In other words, whatever the problems you are facing, somebody, somewhere, had already solved them. Creative problem solution involved finding the solution and adapting it to your problems.
TRIZ was most useful in roles such as product development, design engineering, and process management. For example, Six Sigma quality improvement processes often made use of TRIZ. TRIZ theory was often accepted as a problemsolving analysis and forecast tool derived from the study of patterns of invention in the global patent literature. TRIZ theory consisted of 840 principles of invention, a table of 39 contradictions, 76 standard solutions, 8 rules of technological evolution systems, the final IFR ideal solution, and so on [1].

1.2. The Key TRIZ Tools and Steps. Let us look at two of the central concepts behind TRIZ: generalizing problems and solutions and eliminating contradictions.

1.2.1. Generalizing Problems and Solutions. The primary findings of TRIZ research were as follows: 
(i) Problems and solutions were repeated across industries and sciences. By representing a problem as a "contradiction" (we explored this later in this article), you could predict creative solutions to that problem. Patterns of technical evolution tended to repeat themselves across industries and sciences.

(ii) Creative innovations often used scientific effects outside the field where they were developed. Using TRIZ consisted of learning these repeating patterns of problems and solutions, understanding the contradictions present in a situation, and developing new methods of using scientific effects. You then applied the general TRIZ patterns to the specific situation that confronted you and discovered a generalized version of the problem.

Using Figure 1 you might take the specific problems that you faced and generalize them to one of the TRIZ general problems. From the TRIZ general problems, you identified the general TRIZ solution you needed and then considered how you could apply it to your specific problems. The TRIZ databases were actually a collection of "open source" resources compiled by users and aficionados of the system (such as the 40 principles and 76 standard solutions).

\subsubsection{Eliminating Contradictions. Another fundamental} TRIZ concept was that there were fundamental contradictions at the root of most problems. In many cases, a reliable way to solve a problem was to eliminate these contradictions. TRIZ recognized two categories of contradictions and technical contradictions. These were classical engineering "trade-offs," where you could not reach the desired state because something else in the system prevented it. In other words, when something got better, something else automatically got worse. For example, the product got stronger (good) but the weight increased (bad). Service was customized to each customer (good), but the service delivery system got complicated (bad). Training was comprehensive (good), but it kept employees away from their assignments (bad). The key technical contradictions were summarized in the TRIZ contradiction matrix. As with all TRIZ resources, it takes time and study to become familiar with the contradiction matrix physical (or "inherent") contradictions. These were situations in which an object or system suffers contradictory, opposite requirements. The opposite requirements could be imported into the matrix to deduce the novelty schemes for designing new products. Everyday examples included software that should be complex (to have many features) but also simple (to be easy to learn). All examples above showed us different requirements for products which could enable us to use matrix to deduce the design for products. You could solve physical contradictions with the TRIZ separation principles. These separated your requirements according to basic categories of space, time, and scale.

1.2.3. Key Points. TRIZ was a system of creative problem solving, commonly used in engineering and process management. It followed four basic steps: (i) Defined your specific problems.

(ii) Find the TRIZ generalized problems which match them.

(iii) Find the generalized solution that solves the generalized problems.

(iv) Adapt the generalized solution to solve your specific problems.

Most problems stemmed from technical or physical contradictions. After applying one of hundreds of TRIZ principles and laws to eliminate these contradictions, you could solve the problems.

\section{Background}

The latest national cancer report in January 2019, national cancer statistics, was released by the National Cancer Center. Malignant tumors (cancers) have become one of the major public health problems that seriously threaten the health of the Chinese population. Malignant tumors accounted for the majority of deaths. $23.91 \%$ of the total deaths were caused by malignant tumors, and the incidence and mortality of malignant tumors have been rising continuously in the recent ten years. Malignant tumor occurrence every year would lead medical costs to exceed 220 billion. In 2015, the incidence of malignant tumors was about 3.929 million, and the death rate was about 2.338 million. On average more than 10,000 people were diagnosed with cancer every day. Over the past 10 years, the malignant incidence of female tumors increased by about 3.9\%, and the mortality rate increased by $2.5 \%$ annually. The cancer types of males by order were lung cancers, liver cancers, gastric cancers, esophageal cancers, and colorectal cancers in turn. The top 10 malignant tumors in males accounted for $87.60 \%$ of all malignant tumors in males. Death causes of major malignant tumors in women were lung cancers, gastric cancers, liver cancers, colorectal cancers, and breast cancers, in order. The top 10 malignant tumors in women accounted for about all malignant tumors, $80.50 \%$, of the patients who died of female tumors. At present, the 5-year relative survival rate of malignant tumors in China was increasing to $40.5 \%$, but there was still a big gap with developed countries. It is estimated that, in 2015, the number of new malignant tumors in China was about 3.929 million, including 21.551 million males and 1.788 million females. On average, 7.5 people are diagnosed with cancer every minute $[2,3]$. The incidence of lymphomas in China was about $9 / 100,000$ for males, $8 / 100,000$ for females, and about 2/100,000 for Hodgkin's lymphomas. In different clinical stages of Hodgkin's lymphomas, the 5-year survival rate was $92.5 \%$ in stage I, $86.3 \%$ in stage II, $69.5 \%$ in stage III, and $31.9 \%$ in stage IV. The prognosis of non-Hodgkin's lymphomas was related to pathological types and stages. The 6-year survival rate was $61 \%$ for those with good diffuse lymphocyte differentiation, $42 \%$ for those with poor diffuse lymphocyte differentiation, and only $30 \%$ for those with lymphoblastic lymphomas [4]. The latest national cancer report showed us that the situation of prevention and control was severe. The most schemes of 


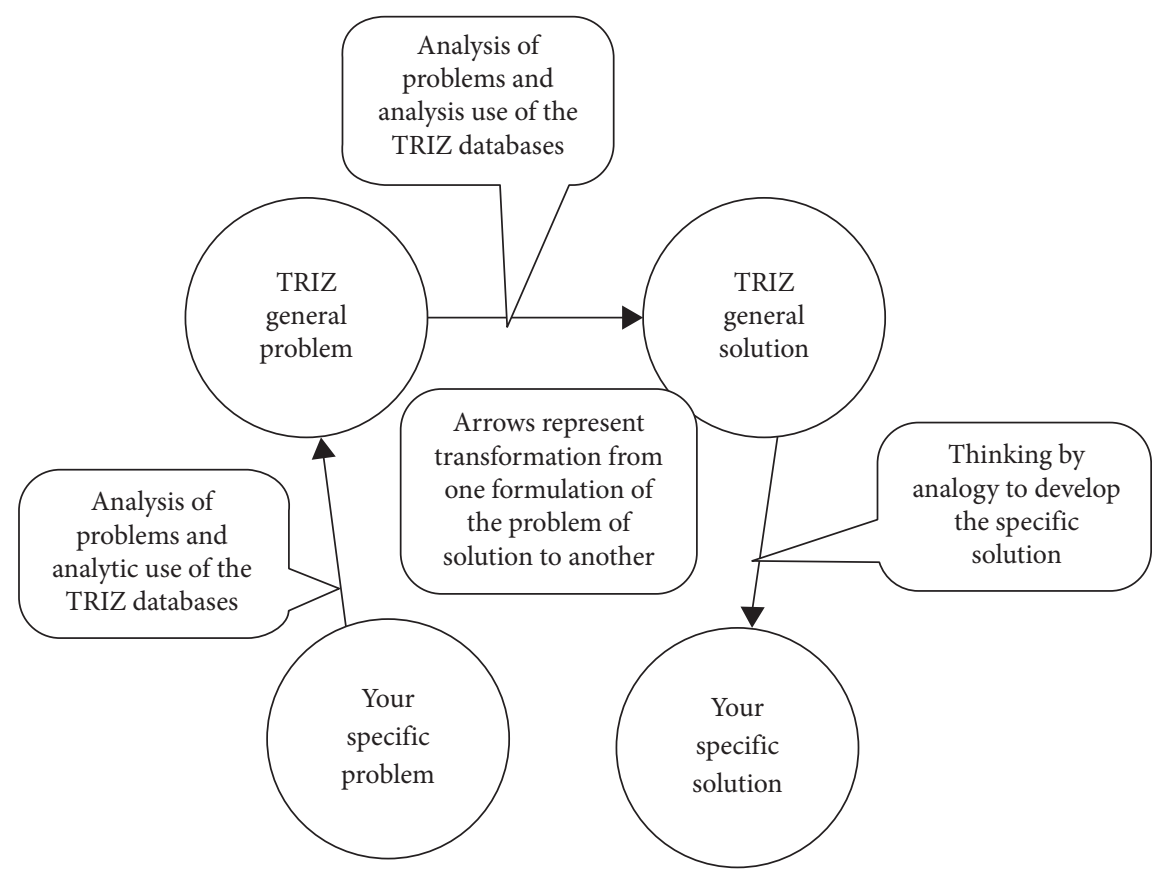

FIGURE 1: The TRIZ problem-solving method.

cancer therapy had their limitation especially when the patients suffered terminal cancers. The low 5-year survival ratio, the high occurrence of malignant tumors, and high costs of cancer therapy always lead to and appeal for the revolution on present conventional therapy for cancers. Many new treatments and researches had been continuously set to try new breakthrough in relative areas. The innovations and their methods for selecting documents to verify research value were very important in cancer treatments. The conventional flow of retrieving information for metaanalyses had its own limitation in control fields and output results. There were also blindness areas to documents distribution in relative fields like authors and keywords. There was no process of visual analysis (bibliometrics) in formulating retrieval strategies at the areas of traditional literature retrieval. There was also no bibliometric analysis to formulate retrieval terms in meta-analyses. Thus, in this article, we combined TRIZ to filter and screen innovation points and visual analysis of bibliometrics to make the innovation of retrieval literature more efficient and reliable.

\section{The Methods of TRIZ and Bibliometrics}

By limiting the retrieval database in the core database, the search strategy was used as "TITLE: (Therap* OR treat*) AND TITLE: (lymph cancer OR lymphadenocarcinoma OR lymphadenoma OR adenolymphoma OR lymph-gland tumor OR lymphoma*) AND TITLE: (magnet* OR electr* OR therm* OR evironmen* OR internal environment OR body fluid)". After retrieving the documents, we exported all those articles into text format. Then, we further analyzed the research status with CiteSpace software using different keywords, regions, countries, schools, and co-citation fields, respectively. We also used the Bibliographic Information
Mining System (BICOMS) to handle the exported documents and export the field matrix including keywords, authors, title, geography, institutes, etc. We then used the Statistical Package for the Social Sciences (SPSS) software to perform the analysis of system cluster. The unified formula of system cluster was developed using Lance and Williams model as follows:

$$
D_{k r}^{2}=\alpha_{p} D_{k p}^{2}+\alpha_{q} D_{k p}^{2}+\beta D_{p q}^{2}+\gamma\left|D_{k p}^{2}-D_{k p}^{2}\right|,
$$

where $\alpha_{p}, \alpha_{q}, \gamma$, and $\beta$ are parameters.

In the next step, we performed two-way cluster analysis using the Gluto software. The formula of two-way cluster was developed using Plaid model as follows:

$$
\left.Y_{i j}=\left(\mu_{0}+\alpha_{i 0}+\beta_{j 0}\right) \sum_{k=1}^{K}\left(\mu_{k}+\alpha_{i k}+\beta_{j k}\right) \rho_{i k} \kappa_{j k}+\varepsilon_{i j}\right) \text {, }
$$

where $\mu, \alpha$, and $\beta$ represent mean values and $\rho$ and $\kappa$ represent a row or layers.

3.1. The Reasoning Process for the Schemes of Finding Novelty in Cancer Research. We used the table of 39 contradictions to give us a thinking path for cancer therapy. TRIZ theory provided us with very different methods from the present general ones of treating cancers which might enlighten us for future research on cancers. We took the steps of using the contradictions matrix to determine possible schemes for treating cancer. Firstly, the popular treating schemes had some effects (killing cancer cells while killing normal cells) on patients' health. The opposite requirements which could be imported into the matrix were side effects and functioning properly. Thus, we chose transverse characteristics (improving factors) as object- 
generated harmful factors (AG 31) and lengthwise characteristics (worsening factors) to ease many factures (32) to form our matrix selection. We ascertained the contradiction matrix as per the following table to determine the schemes for treating cancers.

We listed all schemes from the matrix above in Table 1, respectively, as $21,23,33,1,34,40,11$, and 12 . We compared and reviewed each scheme from the contradiction matrix, respectively, with present popular schemes for treating cancers and tried to provide new views, methods, and thinking paths for treating cancers.

\section{Objective}

Through the use of TRIZ matrix, we could select the possible novelty options for the researches on treating cancers. We then analyzed the various options offered in the matrix by comparing all current treatment options. We classified and analyzed some schemes of cancers in order to find common points including common shortcomings and advantages. We then scanned and filtered the schemes and ideas by principles of TRIZ theory according to innovation points and tried to find out the new measures from TRIZ that differed from present general schemes of cancers. We then analyzed the documents to form the search strategy according to relevant fields of bibliometrics including keywords, subject hotspots, institutions, citations, magazines, etc. Through combining TRIZ theory and bibliometrics, the search strategy for literature on cancer treatment with innovation would be provided, which is shown in Figures 2 and 3.

\section{Results}

5.1. Comparing and Screening Schemes for Innovation in Cancer Research by the Principles of TRIZ Matrix. The content for the schemes of cancer treatment listed above could be explained by the following principles.

5.1.1. Principle 21: Skipping. We might deduce that cancer patients should be checked and diagnosed by regular convention medical checking which might require a lot of time for treating cancers. This was often stressed by the difference of survival ratio of most cases with the length of time ahead for surgical operations. The difference of the survival ratio between US and Chinses cancer patients was partially at the length of time and early check-ups or early therapy. We released chemotherapeutic drugs at lesion areas or released capsules by treating plans and time tables to enhance therapeutic effect for patients. The three main methods for treating cancers were operation, chemotherapy, and radiotherapy by Principle 21, skipping, which could be explained as early as possible after diagnosis. Other therapies such as proton therapy, cryotherapy, laser treatment, electrocautery, heavy ion therapy, and cyberknife could all be classified by this principle.

5.1.2. Principle 23: Feedback. We classified present biological treating and immunotherapy as Principle 23. We knew that the IFN-a, IFN-b, TNF-a, IL-2, G-CSF, GM-CSF, and EPO had been approved or used for treating cancers by NF$\mathrm{kB}$ single path. The nutlins had been used to treat cancers by disturbing the gene regulation of P53 and MDM2. Rapamycin could be used on PC-3 SC by significant growth inhibition with rapamycin. The additive effect of IRS-1 ASO and IHC-20\% could decrease in proliferative index by rapamycin. The recent development of gene treatment of IL2/LAK therapy for refractory acute monoblastic leukemia relapsing has been reported. The CAR-T (chimeric antigen receptor) antibodies Ipilimumab and Tremelimumab have been developed to treat melanoma, kidney cancer, prostate cancer, lung cancer, and other clinical cases. Targeted drugs for lymphomas such as Lenalidomide, Idelalisib, Obinutuzumab, Rituximab, Ibritumomab Tiuxetan, Nivolumab, Pembrolizumab, Bortezomib, Ibrutinib, Lenalidomide, Temsirolimus, and Axicabtagene Ciloleucel have been used in clinical treatment. All the above schemes could be thought of as a feedback principle. There were nearly two thousand targets in the reports about target factors or therapy points as targets.

5.1.3. Principle 33: Homogeneity. This scheme provided us with some novel views for treating cancers. In general expression, cancers were considered "toxicants" by traditional Chinese medicine views, and consequently removal was usually wanted. However, from another perspective, the scheme of inducing some homogeneity into cancer patients' bodies was much different from present treatment schemes. We found some presently accepted treating schemes with this idea from Chinese medicine. As3O2 (arsenic) has been used to treat cancers in traditional Chinses medicine for a long time and is now widely accepted as a very effective medicine for treating leukemia. A report about CTL019 methods treating leukemia and saving the life of a US girl was published by scientists in 2015. This principle of homogeneity might enlighten creative ideas or thinking paths in applying magnetic fields or electric flow to deal with cancer cells or cancerous tissue using Principle 33.

5.1.4. Principle 1: Segmentation. Principle 1 could be used to classify present popular therapy methods immunotherapy and target therapy alongside segmentation. The main cancer therapy of operations, chemotherapy, or radiotherapy could be included as resection of cancer and removal from the body of harmful parts.

5.1.5. Principle 34: Discarding and Recovering. This principle could be used to set a research target for treating cancers with a special novel angle beyond the popular present treatment. We discovered that these schemes are different from traditional methods. Discarding usually was not seen as useful or valuable for treating cancers, but we collected some special cases which showed discarding as a creative new thinking path. We might try to search the cases of selfhealing of cancers. A report was presented where a boy with a tumor in Sichuan province (in Western China) has 
TABLE 1: The contradiction matrix of TRIZ theory.

\begin{tabular}{|c|c|c|c|c|c|c|}
\hline \multirow{2}{*}{$\begin{array}{l}\text { TRIZ } \\
\text { Improving } \\
\text { factors }\end{array}$} & \multicolumn{6}{|c|}{ Worsening factors } \\
\hline & $\mathrm{AB}$ & $\mathrm{AC}$ & $\mathrm{AD}$ & $\mathrm{AE}$ & $\mathrm{AF}$ & AG \\
\hline 22 & $19,3,10,5,28$ & $35,31,40,1,34$ & $37,32,28,26,1$ & $17,3,2,5,9,4,3$ & $2,40,15,19,4$ & $35,40,3,10,25$ \\
\hline 23 & $35,24,10,16,5$ & $5,24,3,15,4,1$ & $24,10,32,7,31$ & $18,31,24,9,7$ & $1,15,24,21,12$ & $24,35,40,1,39$ \\
\hline 24 & $19,9,35,3,21$ & $35,21,24,38,3$ & $35,9,24,7,32$ & $35,13,39,18,2$ & $3,19,15,2,18$ & $35,2,25,22,10$ \\
\hline 25 & $19,1,26,35,17$ & $19,1,35,24,13$ & $32,13,4,1,14$ & $35,28,39,2,9$ & $35,19,40,2,15$ & $35,32,19,3,39$ \\
\hline 26 & $3,14,28,15,24$ & $3,4,31,19,15$ & $3,4,19,15,32$ & $14,9,3,4,1,2,1$ & $35,24,18,28,2$ & $19,15,4,33,3$ \\
\hline 27 & $35,15,2,18,28$ & $19,38,35,27,3$ & $32,40,1,10,3$ & $35,17,7,2,28$ & $13,2,24,35,28$ & $3,1,15,14,29$ \\
\hline 28 & $10,35,28,3,5$ & $5,18,35,19,1$ & $24,28,32,2,26$ & $9,31,14,18,19$ & $1,35,21,18,5$ & $35,24,14,39,2$ \\
\hline 29 & $10,18,7,4,32$ & $35,19,3,2,28$ & $19,10,4,37,1$ & $28,35,14,28,2$ & $2,1,21,19,3,3$ & $4,35,21,24,2$ \\
\hline 30 & $\begin{array}{c}25,22,10,24,26,28 \\
32,23\end{array}$ & $\begin{array}{c}24,34,19,10,7 \\
13\end{array}$ & $\begin{array}{c}24,10,7,25,3,28 \\
2,32\end{array}$ & $2,37,3,10,25$ & $7,1,13,21,35$ & $\begin{array}{c}7,10,31,27,21,40 \\
6\end{array}$ \\
\hline 31 & $35,28,19,25,3$ & $3,15,9,31,35$ & $10,23,2,3,26$ & $3,9,35,14,2,3$ & $5,2,35,19,9,2$ & $28,2,18,29,3$ \\
\hline 32 & $2,10,35,36,21,3$ & $\begin{array}{c}10,18,13,2,21 \\
35,3,1\end{array}$ & $9,16,13,2,4,23$ & $\begin{array}{c}18,35,28,14,3 \\
1,16\end{array}$ & $\begin{array}{c}35,1,2,10,3,19 \\
24,18\end{array}$ & $\begin{array}{c}21,23,33,1,34,40 \\
12,11\end{array}$ \\
\hline
\end{tabular}

discarded the hope for recovery and forced himself to eat mud. This special behavior of madly eating mud helped the child to gradually recover from tumor illness with unknown mechanism. However, this special phenomenon has not been explained convincingly especially when we looked up for novelty on promoting the effectiveness of cancer treatment. We found that some methods of folk therapy usually do not use accepted scientific routes. Some cases might imply or contain some mechanism of new scientific discovery previously unknown or might arouse a new scientific revolution in therapy areas. For example, in Chinese folk sayings, there were many reports of drinking fresh human urine to keep people healthy, which can be included as recovery by the discarding for cancer patients. In these papers, the discarding was mainly in the form of serious prescriptions including components of metabolic products (discarding) such as medicine Wulingzhi and feces. The example above could be explained by the idea that rare elements could be supplied by eating mud which could change the inner physiological electrolyte environment and metabolic microenvironment of human beings.

5.1.6. Principle 40: Composite Materials. This principle made us think of Dr. David Ho and his invention of a cocktail therapy for AIDS by CD8 which could effectively fight HIV. Using this principle, several of the present cancer treating methods might be used in combination. For example, we might combine thermotherapy, chemotherapy, and blood therapy to treat digestive tract cancers at different stages with different composite strategies. We might also apply combined treatment with multiple targets together.

5.1.7. Principle 11: Beforehand Cushioning. This principle could be used to prepare for all kinds of cancer therapies which might increase the treating effect and speed patients' recovery. Cancer patients in China were usually diagnosed by a series of tests, and they then began to accept further treating schemes, mainly involving surgical treatment, chemotherapy, or radiotherapy. However, a belief in traditional Chinses medicine stressed that the patient should be made stronger by taking traditional tonics ahead of treatment with chemotherapy or radiotherapy.

By using this principle, we allowed patients to take thermal conductive agents or heating absorbers to multiply the effect of thermotherapy. Another example of beforehand cushioning (Principle 11) was when Angelina Jolie actively required a mastectomy because she had the BRCA1 gene defect.

5.1.8. Principle 12: Equipotentiality. Principle 12 enabled us to focus on physicotherapeutics. We could reach equipotentiality by using electric field therapy. Thermotherapy was also a treating measure by equipotentiality. Hyperbaric oxygen therapy could also be considered an equipotentiality scheme but there was no consensus about the therapy effect on cancers.

In summary, by the analysis above we knew that most of the principles from TRIZ theory that have been used correspond with all the present schemes or might be in researches listed above. We might focus on the principle which was a minor method for the novelty in possible research on curing cancers, which was Principle 34 from TRIZ, and combine bibliometrics to form strategy for selecting documents for meta-analyses.

5.2. The Rough Retrieval Choice of Research Directions with Innovation. In view of the high mortality of cancers and incidence of lymphomas, we might formulate corresponding retrieval strategies (by the data of national cancer statistics) in relative fields. We now focus on Principle 34 which was a minor method for treating cancers and involves a novel search strategy: (Therap* OR cur* OR recov* OR heal* OR treat*) AND (lymph cancer OR lymphadenocarcinoma OR lymphadenoma OR adenolymphoma OR lymph-gland tumor OR lymphoma*) AND (magnet* OR electr* OR therm* OR evironmen* OR internal environment OR body fluid).

A total of 193 articles (2 from WOS databases) were retrieved using the search strategy above. Then, the exported results of the 193 articles were further analyzed by bioinformatics methods to limit the range of keywords, authors, 
countries, institutes, etc. to ascertain the best search strategy for selecting papers on meta-analyses [5-12].

\subsection{Visual Fields' Election and Search Strategy Revision on} Bibliometrics for Cancer Researches. In order to perform meta-analyses, we used the retrieving strategy outlined in the following sections and exported the retrieved papers. We further analyzed the exported literature using different phrases or keywords such as countries, institutes, and journals. We performed two-way clusters and system clusters to determine the "hot" areas and trends of research on lymphomas. Thus, we further made a search strategy to find novel treatments for lymphomas [13-15].

5.3.1. Keywords Analyses. The graph in Figure 2 was the result of keyword cluster analyses. Table 2 presents a list of the frequency and centrality of keywords.

There was also a list of keyword frequency and centrality in Table 2. Through keyword analyses, the keywords which ranked highest in frequency were MRI, carcinoma, lymphomas, magnetic resonance imaging, diagnosis, radiotherapy, radiation therapy, etc. The main point about lymphomas was the tools of diagnosis, and the main therapy method was radiation. We could also see from word frequency that the focus of most studies was metastatic cancer, non-Hodgkin lymphomas, and lymph node metastasis. Breast cancer and rectal cancer had the highest rankings. In the following position came Sezary syndrome. The words which ranked highest in diagnosis were chemotherapy, position emission tomography, and PET/CT sample biopsy. The words mycosis fungoides had centrality of 0.01 , which suggests that fungal or antifungal related agents had the same effectiveness in the treatment of lymph adenocarcinoma or lymphoid. The retrieval field of keywords could provide a new direction and a new mechanism for the study of drugs for lymphomas.

5.3.2. Author Analyses. From the author's frequency in Table 3, we could see that Eich HT and Li Y were the most active scholars in the field of lymphomas. The frequency values of literature by Eich and Li were both five. The next highest author frequency was that of Elsayad and Ota with four papers each; Bischof, Patti, Moustakis, and Iversen all had three papers. Using the analyses in Figure 3, we knew that Ota published three articles in 2016. The authors who ranked the second position and published two papers were, respectively, Walter in 2015, Huang in 2016, Papaioannou in 2011, Rossi in 2009, Hennenfent in 2017, and Rummeny in 2014. From the table and figure above, we could find that the research on lymphatic cancer was hotter in 2015-2017 than in other years.

5.3.3. Countries Analyses. Table 4 represents the nations of the lymphomas researchers. The ranking from the highest to the lowest by number of documents was the United States, Japan, Germany, China, UK, and Italy. The United States ranked the first with 30 papers mainly published in 2005 .
Japan was listed second with 24, Germany third with 21 papers, and China fourth with 20. British authors had 8 published papers and Italians 4 . This also showed us that the numbers of documents from Sweden, India, Taiwan, and Finland were all low, with 2 each. This clearly showed us that the number of documents was rather concentrated in certain countries especially in the first four countries.

5.3.4. Institutes Analyses. Table 4 and Table 5 demonstrate that the United States, Japan, and Germany ranked the highest three by the number of documents published on lymphomas. China ranked fourth, and Britain, Italy, and Sweden ranked 4 th-6th. In comparison with the table of research institutes, the highest three rankings were those of Yokohama City University, Exeter University, and Oxford University, in order of number of papers.

Japan research institutions on lymphomas were Tokyo Medical and Dental University, Tokyo Women's Medical University, and Ming Chi University of Technology. China institution was Hunan Cancer Hospital. The UK research institutions on lymphomas were University of Oxford, University of Cambridge, University of Sheffield, and Exeter University.

5.3.5. Journal Analyses. Table 6 shows that the journal with the most citations was Radiology, cited 80 times. The second highest number of citations was that of Journal of Clinical Oncology, with 70 citations. In the third position came American Journal Roentgenology with 55 citations. In fourth and fifth position were European Radiology and European Journal of Radiology with 42 and 40 citations, respectively. The International Journal of Radiology Oncology ranked sixth with 39 citations. From the tables above, it could be clearly seen that the hot point for lymphatic cancer was radiotherapy but the journals which ranked the second and the third were comprehensive journals. The journals which ranked second, seventh, and eleventh were all highly influential and famous comprehensive magazines.

5.3.6. Cited References and Author Citation. From Table 7 we could find that the most cited author was Kwee TC, followed in descending order by Koh DM, Harisinghani MG, Cheson BD, Brown G, etc. We might focus on the articles listed ahead and follow the most active researchers in order to grasp the trend and research direction for cancer therapy on lymphomas (on the subtypes of lymphomas by the data of national cancer statistics) $[16,17]$.

5.3.7. Two-Way Cluster and System Cluster for Keywords. Figure 4 shows that the hot spots of research on lymphomas were pararectal lymph node, chronic lymphocytic leukemia, and malignant lymphomas. The most clusters of words in hot spots were diffusion weighted magnetic resonance imaging (MRI), diffusion weighted MR imaging, computed monitoring, apparent diffusion coefficient, whole-body diffusion MRI, biphase pulse, laser microdissection, superparamagnetic iron oxide, segmentation, sentinel node 


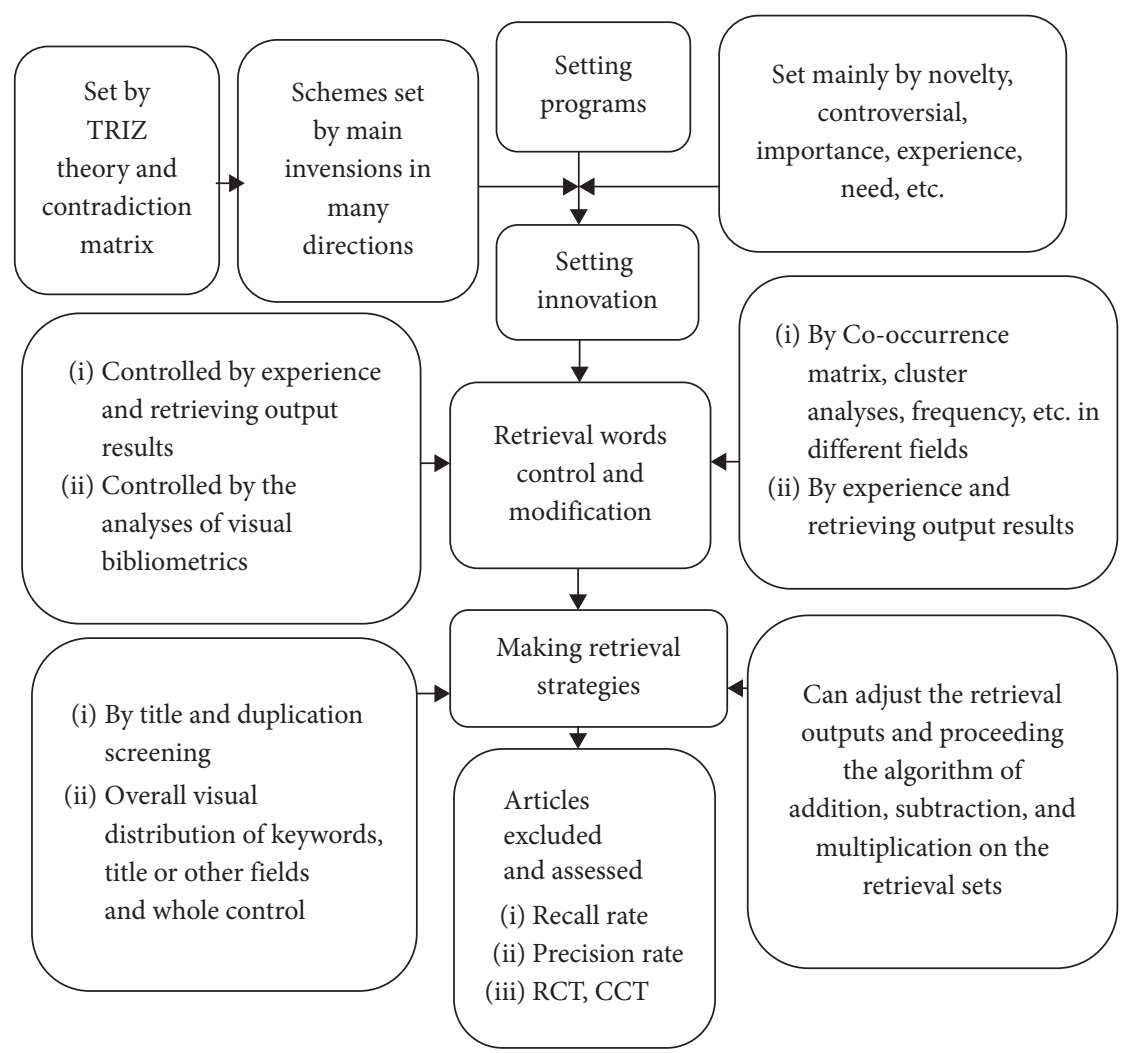

FIgURE 2: The flow of retrieval information for meta-analyses by TRIZ and bibliometrics.

TABle 2: The list of keywords and terms.

\begin{tabular}{lccc}
\hline Count & Centrality & Year & Keywords/noun phrases \\
\hline 34 & 0.48 & 2006 & MRI \\
18 & 0.15 & 2003 & Lymphoma \\
18 & 0.23 & 2006 & Carcinoma \\
13 & 0.02 & 2008 & Magnetic resonance imaging \\
12 & 0.01 & 2005 & Diagnosis \\
9 & 0.04 & 2010 & Radiation therapy \\
9 & 0.2 & 2007 & Radiotherapy \\
9 & 0.2 & 209 & Disease \\
8 & 0.03 & 2007 & Therapy \\
8 & 0.03 & 2011 & Breast cancer \\
7 & .0 .03 & 2005 & Malignant lymphoma \\
7 & 0.05 & 2005 & Lesion \\
7 & 0.12 & 2006 & Metastases \\
7 & 0.05 & 2014 & Tumor \\
7 & 0.23 & 2011 & Biopsy \\
7 & 0.04 & 2013 & Cancer \\
6 & 0.06 & 201 & Dissection \\
6 & 0.03 & 2015 & Metastasis \\
6 & 0.17 & 2012 & Lymph node \\
5 & 0.06 & 2009 & Positron emission tomography \\
5 & 0.01 & 2015 & Sezary syndrome \\
5 & 0.01 & 2015 & Mycosis fungoides \\
4 & 0.00 & 2015 & In vivo \\
4 & 0.00 & 2015 & Rectal cancer \\
4 & 0.01 & 2012 & PET/CT \\
4 & 0.10 & 2005 & Chemotherapy \\
4 & 0.01 & 2016 & Survival \\
\hline & & &
\end{tabular}

navigation, surgery, etc. From the cluster in Figure 5, it could be seen that there were three types of documents in the literature. Among them were three peaks and research hot areas, while other parts were not academic hotspots obviously. The peak boundary of the literature was very mild. The three peaks of literature indicated that the hot spots were not obvious or very sharp. We could see the key points in the literature and judge hot areas of literature by cluster [18-20].

The main means of detection of literature were image retrieval, mainly in chemistry. From the innovative view we might focus on sparse areas of less cluster density such as the signal channel therapy, field therapy, high heat physical therapy, and immune cell therapy which had low literature density.

There was less aggregation of papers in these fields which were not consistent with the current mainstream of treatments. Regarding innovation and diversity, even though the literature above might be seen as cold areas by most people, these papers might represent future novel directions, including the study of cerebral glioma, acute phrase protein, antioxidants, hypoxic condition, carnosol, electrons, and $\mathrm{B}$ cell. Contrary to those very cold areas, the literature on image diagnosis was very hot. We could follow these hot spots for treatment by verifying the evidence-based methods by high density clusters. We could also do research according to the cold distribution especially choosing the direction of low cluster density as an innovative choice for research designs [21-23]. 
TABLE 3: The list of authors.

\begin{tabular}{lcccc}
\hline Order & Authors & Frequency & Ratio & Percentage \\
\hline 1 & Eich, HT & 5 & 0.3566 & 0.566 \\
2 & Li, Y & 5 & 0.3566 & 0.7133 \\
3 & Elsayad, K & 4 & 0.2853 & 0.9986 \\
4 & Ota, M & 4 & 0.2853 & 1.2839 \\
5 & Bischof, M & 3 & 0.2140 & 1.4979 \\
6 & Patti, C & 3 & 0.2140 & 1.7118 \\
7 & Moustakis, C & 3 & 0.2140 & 1.9258 \\
8 & Iversen, L & 3 & 0.2140 & 2.1398 \\
9 & Hennenfent, K & 3 & 0.2140 & 2.3538 \\
10 & Gniadecki, R & 3 & 0.2140 & 2.5679 \\
11 & Meng, Y & 3 & 0.2140 & 2.7817 \\
12 & Galia, M & 3 & 0.2140 & 2.9957 \\
13 & Kamstrup, MR & 3 & 0.2140 & 3.2097 \\
14 & Ugurel, S & 3 & 0.2140 & 3.4237 \\
15 & Galaznik, A & 3 & 0.2140 & 3.6377 \\
16 & Brocker, EB & 3 & 0.2140 & 3.8516 \\
17 & Ike, H & 3 & 0.2140 & 4.0656 \\
18 & Kinugasa, T & 3 & 0.2140 & 4.2796 \\
19 & Sugihara, K & 3 & 0.2140 & 4.4936 \\
20 & Becker, JC & 3 & 0.2140 & 4.7076 \\
21 & Bell, J & 3 & 0.2140 & 4.9215 \\
22 & Haverkamp, U & 3 & 0.2140 & 5.1355 \\
23 & Mule, A & 3 & 0.2140 & 5.3495 \\
24 & Debus, J & 3 & 0.2140 & 5.5635 \\
25 & Brualla, L & 3 & 0.2140 & 5.7775 \\
26 & Sauerwein, W & 3 & 0.2140 & 5.9914 \\
27 & Albano, D & 3 & 0.2140 & 6.2054 \\
28 & Eadd, M & 3 & 0.2140 & 6.4194 \\
29 & Shou, Y & 3 & 0.2140 & 6.6334 \\
30 & Specht, L & 3 & 0.2140 & 6.8474 \\
\hline & & & &
\end{tabular}

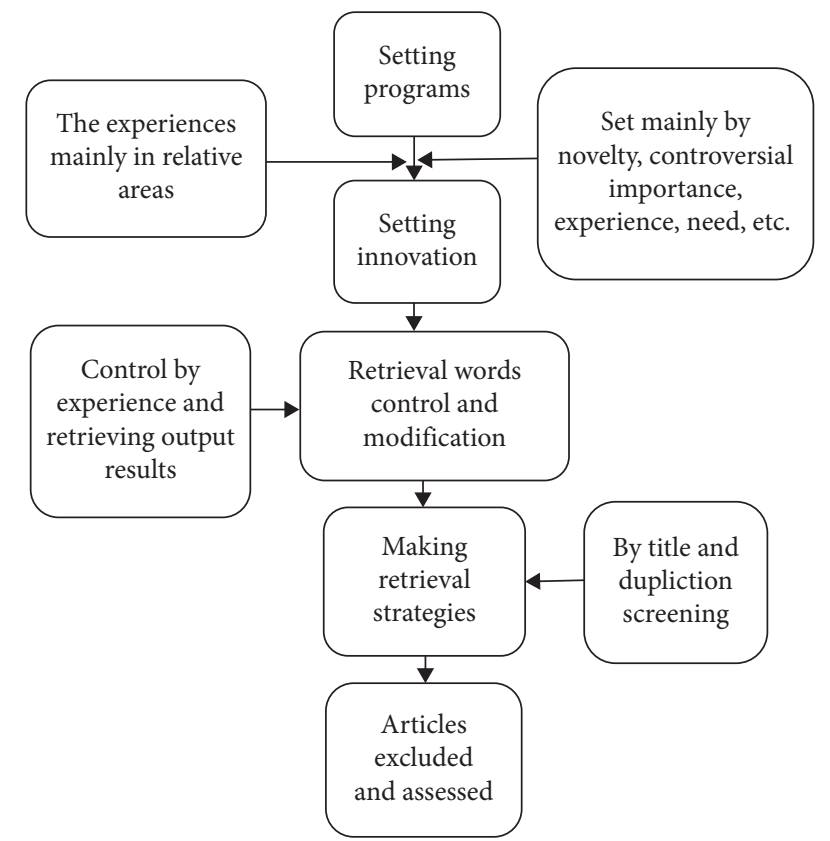

Figure 3: The conventional flow of retrieval information for metaanalyses.
TABLE 4: The list of countries.

\begin{tabular}{lccc}
\hline Count & Centrality & Year & Countries \\
\hline 30 & 0.09 & 2005 & USA \\
24 & 0.00 & 2005 & Japan \\
21 & 0.00 & 2003 & Germany \\
20 & 0.00 & 2010 & China \\
8 & 0.15 & 2011 & England \\
4 & 0.00 & 2009 & Italy \\
2 & 0.00 & 2017 & Sweden \\
2 & 0.00 & 2009 & Finland \\
2 & 0.00 & 2017 & Taiwan \\
2 & 0.00 & 2012 & France \\
2 & 0.00 & 2011 & Denmark \\
2 & 0.00 & 2015 & Wales \\
2 & 0.00 & 2016 & India \\
\hline
\end{tabular}

TABle 5: The list of institutions.

\begin{tabular}{lccc}
\hline Count & Centrality & Year & Institutions \\
\hline 3 & 0 & 2016 & Yokohama City University \\
2 & 0 & 2015 & University of Exeter \\
2 & 0 & 2015 & University of Oxford \\
2 & 0 & 2009 & Tampere University of Technology \\
2 & 0 & 2009 & Essen University Hospital \\
2 & 0 & 2016 & Tokyo Medical and Dental University \\
2 & 0 & 2017 & Ming Chi University Hospital \\
2 & 0 & 2017 & Chettinad Academy of Research and \\
2 & 0 & 2009 & Muenster University Hospital \\
2 & 0 & 2016 & Tokyo Women's Medical University \\
2 & 0 & 2017 & Saiseikai Yokohamashi Nanbu Hospital \\
2 & 0 & 2016 & Hunan Cancer Hospital \\
2 & 0 & 2016 & Madurai Kamaraj University \\
2 & 0 & 2016 & University of Cambridge \\
2 & 0 & 2016 & National Defense Medical College \\
2 & 0 & 2015 & Millennium Pharmaceuticals, Inc. \\
2 & 0 & 2016 & University of Sheffield \\
2 & 0 & 2017 & Karolinska University Hospital \\
2 & 0 & 2011 & Central University \\
2 & 0 & 2009 & Copenhagen University \\
2 & 0 & 2016 & Technical University of Munich \\
2 & 0 & 2011 & Tampere University \\
2 & 0 & 2014 & Bangor University \\
2 & 0 & 2009 & Kindai University \\
2 & 0 & 2015 & Kurume University \\
\hline
\end{tabular}

From the analyses of different phrases above, we might choose the proper words for a retrieving strategy. We might choose hot words for treating schemes and cold words for research with novelty. We might also limit the retrieving areas to select papers for meta-analyses [24-26]. We might limit the geographic area such as the US, Japan, Germany, China, and England by the nations corresponding to analyses. We might also select from a limited range of journals such as Radiology, Journal of Clinical Oncology, American Journal of Roentgenology, European Radiology, European Journal Radiative Oncology, Cancer, New England Journal of 
TABLE 6: The list of journals.

\begin{tabular}{|c|c|c|c|}
\hline Count & Centrality & Year & Cited Journals \\
\hline 80 & 0.13 & 2003 & Radiology \\
\hline 70 & 0.16 & 2003 & J Clin Oncol \\
\hline 55 & 0.13 & 2003 & Am J Roentgenol \\
\hline 42 & 0.04 & 2005 & Eur Radiol \\
\hline 40 & 0.16 & 2005 & Eur J Radiol \\
\hline 39 & 0.06 & 2005 & Int J Radiat Oncol \\
\hline 36 & 0.01 & 2005 & Cancer \\
\hline 35 & 0.06 & 2005 & New Engl J Med \\
\hline 34 & 0.28 & 2005 & Blood \\
\hline 33 & 0.01 & 2008 & J Magn Reson Imaging \\
\hline 25 & 0.05 & 2005 & Ann Oncol \\
\hline 18 & 0.03 & 2011 & Ann Surg Oncol \\
\hline 18 & 0.06 & 2009 & Lancet Oncol \\
\hline 17 & 0.07 & 2011 & J Nucl Med \\
\hline 17 & 0.01 & 2005 & Am J Neuroradiol \\
\hline 15 & 0.00 & 2005 & Acta Radiol \\
\hline 14 & 0.01 & 2009 & J Comput Assist Tomo \\
\hline 14 & 0.07 & 2011 & Ann Surg \\
\hline 13 & 0.03 & 2008 & Invest Radiol \\
\hline 13 & 0.02 & 2005 & Proc Natl Acad Sci USA \\
\hline 13 & 0.04 & 2011 & CA Cancer J Clin \\
\hline 13 & 0.06 & 2007 & Magn Reson Med \\
\hline 13 & 0.03 & 2011 & Clin Radiol \\
\hline 13 & 0.02 & 2013 & Clin Cancer Res \\
\hline 12 & 0.06 & 2009 & Eur J Nucl Med Mol \\
\hline 12 & 0.00 & 2016 & PLOS One \\
\hline 12 & 0.12 & 2005 & Cancer Res \\
\hline 12 & 0.06 & 2005 & Brit J Cancer \\
\hline 12 & 0.00 & 2009 & Cancer Imaging \\
\hline
\end{tabular}

Medicine, Blood, Ann Oncology, Surgical Oncology, or Lancet Oncology.

5.4. The Search Strategy and Result. We might choose the cold words for novelty schemes with limitations such as "glioma, acute phrase protein, antioxidants, hypoxic condition, carnosol, electrons, or B Cell." We might also choose the hot words for meta-analyses limited to "perirectal lymph node, chronic lymphocytic leukemia, or malignant lymphomas" or the hot spot of research on T-cell lymphomas. The most clusters of words in hot spots were "diffusion weighted magnetic resonance imaging (MRI), diffusion weighted MR imaging, computed monitoring, apparent diffusion coefficient, whole-body diffusion MRI, biphase pulse, laser microdissection, superparamagnetic iron oxide, segmentation, sentinel node navigation, surgery, etc." Thus, we might select the documents of hot areas with the retrieving strategy and search results as follows [27-29]:

(1) Result: 156

(2) Search strategy: subject: (non-Hodgkin's lymphomas OR Hodgkin's lymphomas OR perirectal lymph node OR chronic lymphocytic leukemia OR malignant lymphomas OR hot spot of the research on T-cell lymphomas OR diffusion weighted magnetic resonance imaging (MRI) OR diffusion
TABLE 7: The list of cited references.

\begin{tabular}{|c|c|c|c|c|}
\hline Order & Cited authors & Frequency & Ratio & Percentage \\
\hline 1 & Kwee, TC & 30 & 0.5730 & 0.5730 \\
\hline 2 & Koh, DM & 18 & 0.3438 & 0.9167 \\
\hline 3 & Harisinghani, MG & 17 & 0.3247 & 1.2414 \\
\hline 4 & [Anonymous] & 16 & 0.3056 & 1.5470 \\
\hline 5 & Cheson, BD & 15 & 0.2665 & 1.8335 \\
\hline 6 & Brown, G & 14 & 0.2674 & 2.1008 \\
\hline 7 & Lin, C & 13 & 0.2483 & 2.3491 \\
\hline 8 & Kamstrup, MR & 12 & 0.2292 & 2.5783 \\
\hline 9 & Willemze, R & 12 & 0.2292 & 2.8075 \\
\hline 10 & Kim, YH & 11 & 0.2292 & 3.0367 \\
\hline 11 & Oshrakh, MI & 11 & 0.2101 & 3.2468 \\
\hline 12 & Vandecaveye, $\mathrm{V}$ & 11 & 0.2101 & 3.4568 \\
\hline 13 & Hoppe, RT & 10 & 0.2101 & 3.6669 \\
\hline 14 & Sumi, M & 10 & 0.1910 & 3.8579 \\
\hline 15 & Cancer Research UK & 9 & 0.1910 & 4.0489 \\
\hline 16 & Thoeny, HC & 9 & 0.1910 & 4.2399 \\
\hline 17 & Veronesi, U & 9 & 0.1719 & 4.4118 \\
\hline 18 & Jones, OW & 8 & 0.1719 & 4.5837 \\
\hline 19 & Yang, $\mathrm{F}$ & 8 & 0.1719 & 4.7555 \\
\hline 20 & Elsayad, K & 8 & 0.1719 & 4.9274 \\
\hline 21 & Olsen, E & 8 & 0.1528 & 5.0802 \\
\hline 22 & Shaikh, SR & 8 & 0.1528 & 5.2330 \\
\hline 23 & Harrison, C & 8 & 0.1528 & 5.3858 \\
\hline 24 & $\mathrm{Wu}, \mathrm{XC}$ & 8 & 0.1528 & 5.5386 \\
\hline 25 & Navi, D & 8 & 0.1528 & 5.6914 \\
\hline 26 & $\mathrm{Kim}, \mathrm{CK}$ & 8 & 0.1528 & 5.8442 \\
\hline 27 & Choi, HJ & 8 & 0.1528 & 5.9969 \\
\hline 28 & Duvic, $\mathrm{M}$ & 8 & 0.1528 & 6.1497 \\
\hline 29 & Toh, CH & 7 & 0.1337 & 6.2834 \\
\hline 30 & Haldorsen, IS & 7 & 0.1337 & 6.4171 \\
\hline 31 & Weissleder, R & 7 & 0.1337 & 6.5508 \\
\hline 32 & Jones, GW & 7 & 0.1337 & 6.6845 \\
\hline 33 & Hoppe, RT & 7 & 0.1337 & 6.8182 \\
\hline 34 & Wilson, LD & 7 & 0.1337 & 6.9519 \\
\hline 35 & $\mathrm{Ng}, \mathrm{AD}$ & 7 & 0.1337 & 7.0856 \\
\hline 36 & King, $A D$ & 7 & 0.1337 & 7.2193 \\
\hline 37 & Winter, A & 7 & 0.1337 & 7.3529 \\
\hline 38 & Akiyoshi, T & 7 & 0.1337 & 7.4866 \\
\hline 39 & Briganti, A & 7 & 0.1337 & 7.6203 \\
\hline 40 & Cha, S & 7 & 0.1337 & 7.7540 \\
\hline
\end{tabular}

weight MR imaging OR computed monitoring OR apparent diffusion coefficient OR whole-body diffusion MRI OR biphase pulse OR laser microdissection OR superparamagnetic iron oxide OR segmentation OR sentinel node navigation OR surgery) AND subject: (lymph cancer OR lymphadenocarcinoma OR lymphadenoma OR adenolymphoma OR lymph-gland tumor $\mathrm{OR}$ lymphoma*) AND subject: (Therap* OR cur* OR recov* OR heal $^{*}$ OR treat*) AND subject: (magnet* OR electr* OR therm* OR evironmen* OR internal environment OR body fluid) AND address: (US OR China OR Japan OR Germany OR England) AND publication source: (Radiology OR J Clin Oncol OR Am J Roentgenol OR Eur Radiol OR Eur J Radiol OR Cancer OR New Engl J Med OR Blood OR Ann Oncol OR Ann Surg Oncol OR Lancet Oncol) [156 results]. 


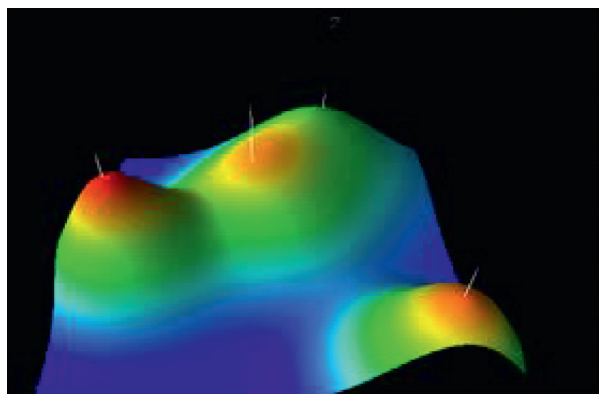

Figure 4: System cluster of keywords.
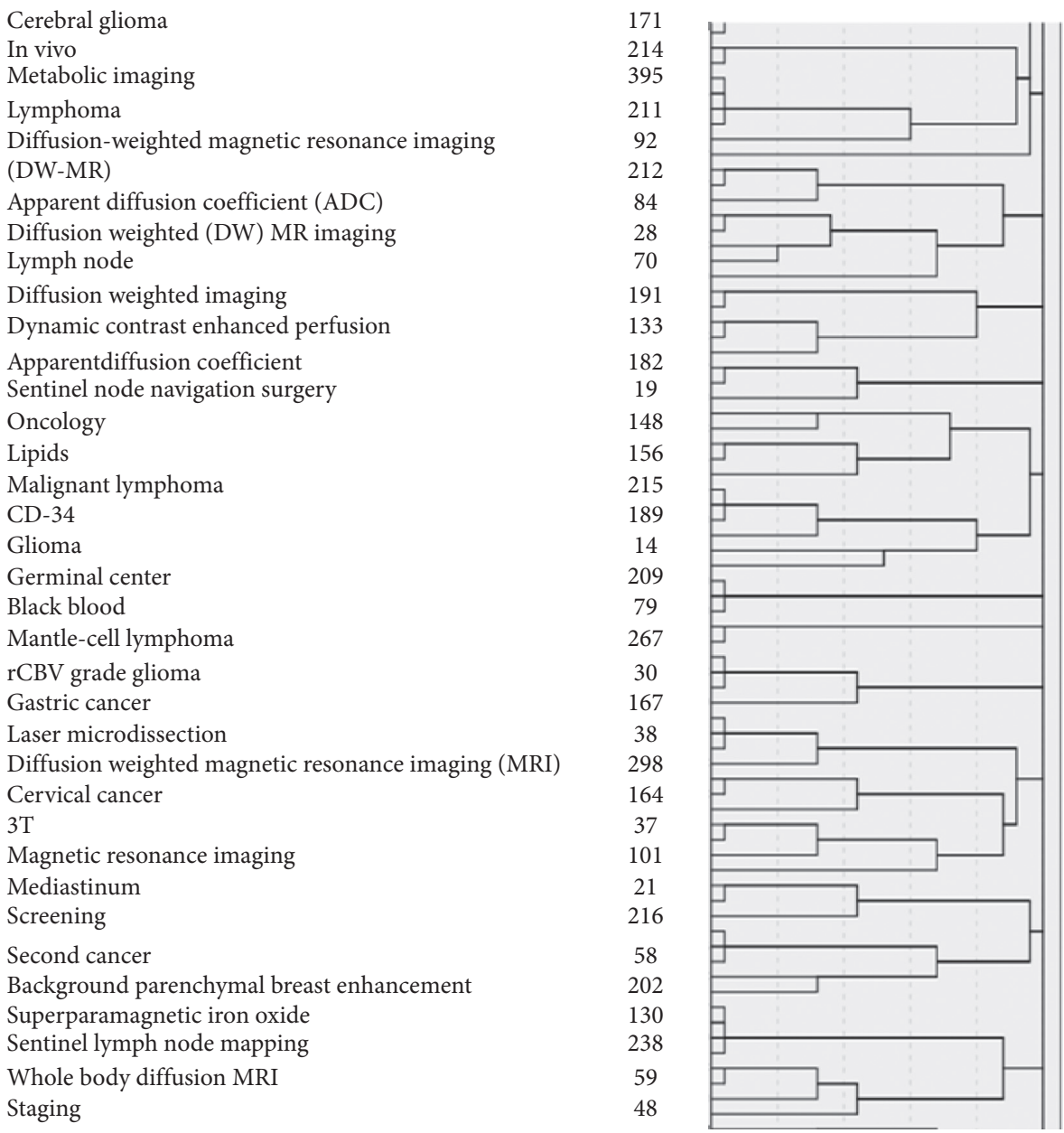

Figure 5: Two-way cluster of keywords.

Thus we might select the documents of cold areas with the retrieving strategy and search result as follows:

(1) Result: 184 (from WOS databases)

(2) Search strategy: subject: (glioma OR acute phrase protein $\mathrm{OR}$ antioxidants $\mathrm{OR}$ hypoxic condition $\mathrm{OR}$ carnosol OR electrons OR B Cell) AND subject: (lymph cancer OR lymphadenocarcinoma OR lymphadenoma OR adenolymphoma OR lymphgland tumor OR lymphomas or non-Hodgkin's lymphomas or Hodgkin's lymphomas) AND subject: (Therap* OR cur* OR recov* OR heal* OR treat*)
AND subject: (magnet* OR electr* OR therm* OR evironmen* OR Internal environment OR body fluid) AND address: (US OR China OR Japan OR Germany OR England) AND publication source: (Radiology OR J Clin Oncol OR Am J Roentgenol OR Eur Radiol OR Eur J Radiol OR Cancer OR New Engl J Med OR Blood OR Ann Oncol OR Ann Surg Oncol OR Lancet Oncol) [184 results].

(1) Result: 106 (from WOS databases)

(2) Search strategy: (canci* OR cancer OR tumor* OR onclog* OR lymph cancer OR lymphadenocarcinoma 
OR lymphadenoma OR adenolymphoma OR lymphgland tumor OR lymphoma*) AND (cur* or recov* or kill* or heal* or treat* or Therapeutic* or therap*) AND (urine OR waste OR urine OR defecate OR shit OR dung OR cow dung OR camel urine OR cat feces coffee OR civet coffee OR disinfection urine OR urea or uric acid).

(1) Result: 15 (from WOS databases and CNKI databases)

(2) Search strategy: (Wulingzhi OR Mochizuki sand OR night sand OR ambergris OR white cloves OR chicken vera OR white lilac OR silkworm OR insect tea OR cow dung OR camel urine OR cat feces coffee OR civet coffee OR disinfection urine OR stool Or urea or uric acid OR muscle liver OR hippuric acid OR oxalic acid OR urine blue mother) AND (canci* OR cancer OR tumor* OR lymph cancer OR lymphadenocarcinoma OR lymphadenoma OR adenolymphoma OR lymph-gland tumor OR lymphoma*) AND (cur* OR recov* OR kill* OR treat*) [15 results].

As a comparison, we could see that the number and domain of retrieval documents cannot be controlled without the modification by bibliometric analysis. The distribution of their various subfields of retrieval output could not be known by conventional meta-analyses. The following were the retrieval results by conventional meta-analyses without modification by bibliometrics (with title field).

(1) Result: 2166 (from WOS databases)

(2) Search strategy: Title: (non-Hodgkin's lymphomas OR Hodgkin's lymphomas OR perirectal lymph node OR Chronic lymphocytic leukemia OR malignant lymphomas OR hot spot of the research on T-cell lymphomas OR diffusion weighted magnetic resonance imaging (MRI) OR diffusion weight $M R$ imaging OR computed monitoring OR apparent diffusion coefficient OR whole-body diffusion MRI OR biphase pulse OR laser microdissection OR superparamagnetic iron oxide OR segmentation OR sentinel node navigation OR surgery) AND subject: (lymph cancer OR lymphadenocarcinoma OR lymphadenoma OR adenolymphoma OR lymphgland tumor OR lymphoma*) AND subject: (Therap* OR cur* OR recov* OR heal* OR treat*) [2166]

\section{Conclusion}

This article tried to apply new method for novelty on doing research by TRIZ and combine bibliometrics to visualize the retrieving process. Using TRIZ theory we could compare the schemes of conventional therapy and select the innovation in cancer researches. Then we could add the idea to the searching strategy to select documents. Using bibliometrics, we could freely select the words for searching strategy by the graph of system cluster and two-way cluster of keywords. We could also know all the hot areas of research and their tendency or distribution. At the same time, the analyses of bibliometrics could also provide us with tools and total vision on keywords distribution, units distribution, journal distribution, author distribution, etc. Thus, we could collect all the possible synonyms and high frequency words to construct search strategy and also ensured precision rate and recall rate, which were very important factors in bibliometrics for searching literature. We could flexibly adjust the search strategy to meet the search objectives, whether they were on research or on clinical treatment, by visualizing any field and setting the threshold for any field. Thus we could make our schemes of researches on cancers more novel and make our schemes of clinical treatment more effective.

In summary, we could reason out novelty from Principle 34 of TRIZ and focus on this principle to form strategy by combining bibliometrics. This article could provide a new method of the application of the interdisciplinary of bibliometrics, TRIZ, and meta-analysis and visualize the retrieving process. The interdisciplinary application above would offer the possibility of freely choosing search strategy in any fields with controlled fields and direction for documents on cancer treatment $[30,31]$.

\section{Data Availability}

The data used to support the findings of this study are included within the article.

\section{Conflicts of Interest}

The authors declare that there are no conflicts of interest regarding the publication of this paper.

\section{Acknowledgments}

This work was supported by MOE (Ministry of Education in China) Project of Humanities and Social Sciences (No.19YJC630116), Medical and Health Data Extraction and Evaluation for Meta-Analysis under Big Data Environment, and Major Project for Science and Technology Innovation Strategy and Soft Science Research in Anhui Province (No. 201806A02020005).

\section{References}

[1] “TRIZ”[OL], https://baike.baidu.com/item/triz/3516799?fr= aladdin.

[2] "The latest issue of National Cancer Center: National Cancer Report 2019”[OL] http://www.sohu.com/a/296354370_ 707276.

[3] "According to the Latest Cancer Data in 2019, 3.93 Million New Cancers Are Reported Every Year, with 7.5 Cancers Per Minute."[OL] http://www.sohu.com/a/292526227_120047572.

[4] "A Comparative Analysis of the Incidence and Survival Rates of Lymphoma between China and the United States"[OL] http://cancer.39.net/a/140312/4353102.html.

[5] Z. Y. Pu, S. X. Jin, and M. Tao, "Treatment of 92 cases of advanced gastric cancer with traditional Chinese medicine combined with chemotherapy," Guangming Chinese Medicine, vol. 28, no. 11, pp. 2359-2360, 2013. 
[6] J. Wang and Y. L. Ren, "Experimental study on the inhibition of proliferation and induction of A549 human lung adenocarcinoma cells by Wulingzhi mixture," Chinese Journal of Chinese Materia Medica, vol. 31, no. 7, pp. 585-587, 2006.

[7] S. M. Chen, D. J. Wang, and M. X. Huang, "22 cases of precancerous lesion of gastric cancer treated with Ping Wei Decoction," Fujian Journal of TCM, vol. 28, no. 3, p. 21, 1997.

[8] J. B. Yuan, "Interventional therapy combined with Fuzheng soup in the treatment of 24 cases of liver cancer," New Journal of Traditional Chinese Medicine, vol. 37, no. 3, pp. 63-65, 2005.

[9] Z. Z. Tang and C. Y. Sun, "Therapeutic effect of Rebirth oral liquid on HBV related liver cancer," Chinese Traditional Patent Medicine, vol. 33, no. 6, pp. 929-931, 2011.

[10] M. Y. Wang and Q. T. Chen, "Randomized parallel controlled study of the combination of Shixiao Powder with three steps for analgesic, omitting, and stasis type of advanced cancer pain," Journal of Practical Traditional Chinese Internal Medicine, vol. 27, no. 6, pp. 79-80, 2013.

[11] S. J. Yang, "Randomized parallel controlled study of syndrome differentiation combined with western medicine in the treatment of advanced gastric cancer," Journal Practical Traditional Chinese Internal Medicine, vol. 29, no. 6, pp. 99-101, 2015.

[12] L. B. Zhu, L. R. Li, Y. S. Li, J. Wang, and Q. Wang, "Chinese herbal medicine as an adjunctive therapy for breast cancer: a systematic review and meta-analysis," Evidence-based Complementary and Alternative Medicine, vol. 2016, Article ID 9469276, 17 pages, 2016.

[13] G. W. Gu and X.-C. Li, "Meta-analysis of the efficacy of transcatheter arterial chemoembolization combined with percutaneous ethanol injection in treating unresectable primary liver cancer," Zhonghua Yi Xue Za Zhi, vol. 89, no. 12, pp. 805-809, 2009.

[14] Q. Wang, X. R. He, J. H. Tian et al., "A meta analysis of aidi injection plus taxotere and cisplatin in the treatment of nonsmall cell lung cancer," Chinese Journal of Lung Cancer, vol. 13, no. 11, pp. 1027-1034, 2010.

[15] R. Z. Xu, L. B. Lin, Y. Li, and Y. Li, "ShenQi FuZheng Injection combined with chemotherapy in the treatment of colorectal cancer: a meta-analysis," Plos One, vol. 12, no. 9, 2017.

[16] M. A. Kharfan-Dabaja, T. Reljic, J. El-Asmar et al., "Reducedintensity or myeloablative allogeneic hematopoietic cell transplantation for mantle cell lymphoma: a systematic review," Future Oncology, vol. 12, no. 22, pp. 2631-2642, 2016.

[17] J. Wei, J. Xu, Y. Cao, J. Zhou, and Y. Zhang, "Allogeneic stemcell transplantation for peripheral T-cell lymphoma: a systemic review and meta-analysis," ACTA Haematologica, vol. 133, no. 2, pp. 136-144, 2015.

[18] K. Fernandes, J. S. Cardoso, and J. Fernandes, "Automated methods for the decision support of cervical cancer screening using digital colposcopies," IEEE Access, vol. 6, pp. 3391033927, 2018

[19] M. Sajjad, S. Khan, Z. Jan et al., "Leukocytes classification and segmentation in microscopic blood smear: a resource-aware healthcare service in smart cities," IEEE Access, vol. 5, pp. 3475-3489, 2017.

[20] A. Kwok, T. Lam, P. Katelaris, and R. W. Leong, "Helicobacter pylorieradication therapy: indications, efficacy and safety," Expert Opinion on Drug Safety, vol. 7, no. 3, pp. 271-281, 2008.

[21] P. J. Brockelmann, D. A. Eichenauer, T. Jakob, M. Follmann, A. Engert, and N. Skoetz, "Hodgkin lymphoma in adults," Deutsches Arzteblatt International, vol. 115, no. 31-32, pp. 535-539, 2018.
[22] L. Specht, "Very long-term follow-up of the Danish National Hodgkin S Group's randomized trial of radiotherapy (RT) alone vs. combined modality treatment (CMT) for early stage Hodgkin lymphoma, with special reference to second tumours and overall survival," Blood, vol. 102, no. 11, pp. 635A-637A, 2003.

[23] J.-Q. Huang and R. H. Hunt, "Review: eradication of Helicobacter pylori. Problems and recommendations," Journal of Gastroenterology and Hepatology, vol. 12, no. 8, pp. 590-598, 1997.

[24] L. Brandt, E. Kimby, P. Nygren, and B. Glimelius, "A systematic overview of chemotherapy effects in Hodgkin's disease," Acta Oncologica (Stockholm, Sweden), vol. 40, no. 2-3, pp. 185-197, 2001.

[25] A. Colosia, P. C. Trask, R. Olivares et al., "Efficacy and safety of treatments for relapsed or refractory DLBCL: results of a systematic literature review," Blood, vol. 122, no. 21, 2013.

[26] G. H. Lyman and N. M. Kuderer, "Gene expression profile signatures to predict survival in diffuse large B-cell lymphoma: a meta-analysis of early results," Blood, vol. 104, no. 11, pp. 624A-626A, 2004.

[27] M. T. Sickinger, B. Von Tresckow, C. Kobe, A. Engert, P. Borchmann, and N. Skoetz, "Positron emission tomography-adapted therapy for first-line treatment in individuals with Hodgkin lymphoma," The Cochrane Database of Systematic Reviews, vol. 1, no. 10, pp. 533-537, 2015.

[28] Z. Si, H. Yu, and Z. Ma, "Learning deep features for DNA methylation data analysis," IEEE Access, vol. 4, pp. 2732-2737, 2016.

[29] J. B. Spinelli, H. Yoon, A. E. Ringel, S. Jeanfavre, C. B. Clish, and M. C. Haigis, "Metabolic recycling of ammonia via glutamate dehydrogenase supports breast cancer biomass," Science, vol. 358, no. 6365, pp. 941-946, 2017.

[30] J.-B. Liu, Y.-S. Ding, Y. Zhang et al., "Anti-inflammatory hydrolyzable tannins fromMyricaria bracteata," Journal of Natural Products, vol. 78, no. 5, pp. 1015-1025, 2015.

[31] L. D. Chong, "Exploiting cancer metabolism," Science, vol. 355, no. 6329, p. 1036, 2017. 